\title{
STUDY THE RELATIONSHIP BETWEEN INTERCELLULAR ADHESION MOLECULES (ICAM), OXIDATIVE STRESS AND INSULIN RESISTANCE AMONG TYPE 2 DIABETIC PATIENTS WITH NEPHROPATHY
}

\author{
El-SaeidMostafa El-Saeid El-Bawab ${ }^{1}$; Nagwa S. Ahmed ${ }^{2}$; LotfyHamed Abu
} Dahab $^{3}$; Mahmoud El-Sayed ${ }^{4}$ and Kenawy El SayedMostafa Kenawy ${ }^{1}$

1,2,4 Medical Biochemistry Department; ${ }^{3}$ Internal Medicine Department ${ }^{2}$ Sohag University, Faculty of medicine, Biochemistry Department, Sohag, Egypt. ${ }^{3}$ Sohag University, Faculty of medicine, Internal Medicine Department, Sohag, Egypt. ${ }^{1}$ Al Azhar University, Faculty of medicine, Assiut Branch, Biochemistry Department, Assiut, Egypt.

${ }^{4}$ Al Azhar University, Faculty of medicine, Assiut Branch, Biochemistry Department, Damietta, Egypt.

\begin{abstract}
BACKGROUND: Diabetes mellitus (DM), especially type 2, represents one of the most important health problems worldwide and, according to recent estimations, it is likely to worsen to critical levels in the next decades, with the great concern that this disease is rising rapidly in younger population groups, including children and adolescents. Oxidative stress is an important factor in the etiology and pathogenesis of diabetes mellitus. Intercellular adhesion molecule-1: Intercellular adhesion molecule1 (ICAM-1), also known as CD54, is an endothelial and leukocyte associated transmembrane protein with relevance in stabilizing cell-cell interactions and facilitating leukocyte endothelial transmigration. It is constitutively present in the membranes of leukocytes and endothelial cells; upon cytokine stimulation, the concentrations greatly increase. ICAM-1 ligation produces pro-inflammatory effects such as inflammatory leukocyte recruitment by signaling through cascades involving a number of kinases.
\end{abstract}

AIM OF THE WORK: In this study, we attempt torelationship between sICAM-1 (intercellular adhesion molecule-1) and diabetic nephropathy as an important marker in inflammation, immune responses and in intracellular signaling events. we determined plasma concentration of ICAM-1, serum malondialdehyde (MDA), superoxide dismutase (SOD), Serum nitric oxide (NO) as a marker for endothelial activation among type- 2 diabetic patients with or without nephropathy and oxidative stress as a marker (as tool in early diagnosis of nephropathy as major diabetic complications) also to explore the relationship between plasma level of ICAM-1 and insulin resistance in the studied patients.

PATIENTS AND METHODS: This study carried out on 100 male individuals. Their age ranged from 25- 60 years. They were classified as follows:Control group: 30 apparently healthy adult males. Patients groups: 70 patients suffering from type 2 diabetes mellitus, Patients were subdivided into two groups. Group I:35 patients with type 2 diabetes mellitus with normal albuminuria without complications.Group II:35 patients with type 2 diabetes mellitus with nephropathy.

RESULTS: This study has found a strong relationship between sICAM-1

(intercellular adhesion molecule-1) and diabetic nephropathy. It has been shown that inflammation has a role in the development of diabetic nephropathy, so sICAM-1 may 
play a role in the development of diabetic nephropathy. Our results also, show strong direct correlation between ICAM-1 and insulin resistance which supports the conclusion that insulin resistance may be a factor contributing to the increase plasma levels of adhesion molecules. In accordance to our results the metabolic syndrome and type 2 diabetes are associated with endothelial activation, but the mechanisms that underlie these associations are not fully understood.

\section{INTRODUCTION}

Diabetes mellitus type 2 is a long term metabolic disorder that is characterized by high blood sugar, insulin resistance, and relative lack of insulin (1). Common symptoms include increased thirst, frequent urination, and unexplained weight loss. Symptoms may also include increased hunger, feeling tired, and sores that do not heal (2). Often symptoms come on slowly (1). Long-term complications from high blood sugar include heart disease, strokes, diabetic retinopathy which can result in blindness, kidney failure, and poor blood flow in the limbs which may lead to amputations (3). The sudden onset of hyperosmolar hyperglycemic state may occur; however, ketoacidosis is uncommon (4).

According to data from the International Diabetes Federation, the number of diabetics older than twenty will rise from 285 million in 2010 to 439 million in 2030. Therefore, target organ complications secondary to diabetes, especially micro and macro vascular complications will be one of the most important medical concerns in the near future. Because of this, a growing number of researches have focused on diabetes and its complications, with the aim to expand our knowledge about pathogenic and pathophysiological mechanisms, preventive strategies and potential novel therapies.

Diabetic nephropathy (DN) is one of the most relevant diabetic complications. In the last decade, DN has become the main cause of endstage renal disease (ESRD) in the Western world, with estimations indicating that type 2 diabetes contributes to a great proportion of patients in renal replacement therapy programs (5). However, this situation is starting to change. While in the general population the incidence of ESRD rises continuously due to the increased prevalence of diabetes mellitus, a recent study found that diabetes-related ESRD incidence in the population $n$ with diabetes has shown a declining trend, suggesting that current efforts in the prevention of ESRD may be successful (6).

Insulin resistance and relative insulin deficiency play key roles in the development of type 2 diabetes (7). Hyperglycemia occurring as result of these factors is critical in the genesis of diabetic complications. Poor glycemic control has been demonstrated as an independent predictor of the development and progression of $\mathrm{DN}$ (8). Although the intimate mechanisms by which hyperglycemia leads to renal injury are not completely known.

Oxidative stress is caused by an imbalance between the production of oxidants or ROS and the capacity of a biological system to readily detoxify the reactive intermediates or repair the resulting damage. The final result is the oxidation of important macromolecules, including proteins, lipids, carbohydrates and DNA. Growing evidence indicates that oxidative stress plays a pivotal role in the development of both micro and macro vascular diabetic complications (9).

Oxidative stress is an important factor in the etiology and pathogenesis of diabetes mellitus (10). 
Cell adhesion molecules are proteins located on the cell surface involved in the binding with other cells or with the extracellular matrix. These proteins are typically transmembrane receptors composed of three domains: an intracellular domain that interacts with the cytoskeleton, a transmembrane domain, and an extracellular domain that interacts either with other adhesion molecules of the same kind (homophilic binding) or different kind or the extracellular matrix (heterophilic binding). Four protein groups are the most important families of cell adhesion molecules: the immunoglobulin superfamily, the integrins, the cadherins and the selectins.

Intercellular adhesion molecule-1: Intercellular adhesion molecule-1 (ICAM-1), also known as CD54, is an endothelial and leukocyte associated transmembrane protein with relevance in stabilizing cell-cell interactions and facilitating leukocyte endothelial transmigration. It is constitutively present in the membranes of leukocytes and endothelial cells; upon cytokine stimulation, the concentrations greatly increase. ICAM-1 ligation produces proinflammatory effects such as inflammatory leukocyte recruitment by signaling through cascades involving a number of kinases (11).

This adhesion molecule is involved in the pathogenesis of diabetic kidney disease (12).

It has been suggested that modulation of ICAM-1 activity (blockade of receptor activation or reduction of expression) may be a therapeutic approach in DN. In a recent experimental study, colchicine administration to streptozotocininduced diabetic rats significantly reduced UAE, inflammatory cell infiltration and extracellular matrix accumulation. These beneficial effects were associated with inhibition of inflammatory molecules expression in the renal tissue, including ICAM-1 (13).

The aim of the present study is to determine plasma concentration of ICAM-1, serum malondialdehyde (MDA), superoxide dismutase (SOD), Serum nitric oxide (NO) as a marker for endothelial activation among type- 2 diabetic patients with or without nephropathy and oxidative stress as a marker (as tool in early diagnosis of nephropathy as major diabetic complications) also to explore the relationship between plasma level of ICAM-1 and insulin resistance in the studied patients.

\section{PATIENTS AND METHODS}

This study carried out on 100 male individuals. Their age ranged from $25-$ 60 years. They were selected from outpatient and inpatient clinics of Sohag University hospital, during the summer months of 2015.They were classified as follows:

Control group: 30 apparently healthy adult males aged 25-65 years served as the control group

Patients groups: 70 patients suffering from type 2 diabetes mellitus, with known hypertension, 16-Shanahan, C.M. (2005): mechanisms of vascular calcification in renal disease. Clin.Nephrol.; 63:146-156.

attended the outpatient and inpatient clinic of the internal medicine department served as our study group. Patients were subdivided into two groups

Group I: 35 patients with type 2 diabetes mellitus with normal albuminuria without complications.

Group II: 35 patients with type 2 diabetes mellitus with nephropathy. An informed written consent was obtained from each patient.

\section{Inclusion criteria:}

We are going to carry out this study on 70 patients suffering from type 2 
diabetes mellitus, which's divided into 35 patients with type 2 diabetes mellitus with normal albuminuria without complications and 35 patients with type 2 diabetes mellitus with nephropathy compared with 30 nondiabetic controls with matched ages.All patients selected were males and should have no liver, or heart diseases.

\section{Methods:}

Sample preparation:

Control and patients should be fasting for 10-14 hours before blood drowned. $10 \mathrm{ml}$ of Fasting blood was (One sample) collected from the healthy adult males and the patients by venipuncture under complete aseptic conditions and then divided into 3 portions one for serum in plain red-top vacutainer tube without additives, and portion with anticoagulant EDTA. Serum samples left to be clotted and then serum was separated by centrifugation at $3000 \mathrm{rpm}$. Serum was separated and divided into separate aliquots and frozen at -70 until assays were performed.

Urine sample also was collected for determination of urine creatinine, urine microalbumen, and for calculation of UACR.

Assays:

Determination Blood concentrations of plasma glucose, total, HDL, LDL cholesterol, triglycerides, creatinine (serum and urine), and blood urea were measured by routine laboratory techniques.

ICAM-ldetermined by ELISA using kits supplied byRayBiotech, IncNorcross, Georgia, USA.

Nitric oxide determined by ELISA using kits supplied byR\&D Systems, Inc. USA.
Hemoglobin $\quad \mathbf{A}_{1}$ Cis determined byImmunoturbidimetric Method using kits supplied by DIATEK Healthcare Pvt. Ltd.

Reduced glutathione (GSH) concentrationis determined by ELISA using kits supplied by The research \& development center of EIAab East Lake Hi-Tech Development Zone, Wuhan China.

Serum insulin is determined by ELISA using kits supplied by Immunospec Corporation Canoga Park, CA, USA.

Superoxide dismutase (SOD)is determined byColorimetric Assayusing kits supplied by Cayman Chemical Company, Ann Arbor, MI, U.S.A

Serum malondialdehyde (MDA) is determined bycolorimetric assay using kits supplied byAbcamInc Kendall Square, Ste 341 Cambridge, MA 02139-1517 USA.

Serum nitric oxide (NO)is determined by ELISA using kits supplied by R\&D Systems, Inc. USA.

Urine microalbumin is determined byQuantitative Latex turbidimetryusing kits supplied bySPINREACT, $\quad$ S.A./S.A.U. Ctra.Santa Coloma, SPAIN.

Urine Albumin/Creatinine

Ratio(UACR)(mg/g creatinine)was calculated from urine micro-albumin and urine creatinine using the equation UACR $=$ (Urine albumin / Urine creatinine) $\mathrm{x} 1000$ and the equation takes albumin in $\mathrm{mg} / \mathrm{dl}$ and creatinine in $\mathrm{mg} / \mathrm{d}$.

Homeostasis model assessment of insulin resistance values were calculated from fasting glucose and insulin measures according to the formula (fasting glucose [mmol/ 1] x fasting insulin $[\mu \mathrm{U} / \mathrm{ml}] / 22.5)$. 


\section{RESULTS}

We measured ICAM-1, insulin, cholesterol, triacylglycerols, HDL-cholesterol, and LDL-cholesterol and creatinine (serum and urine) and urea, glycated hemoglobin, microalbuminuria, reduced glutathione (GSH) concentration, superoxide dismutase (SOD), serum malondialdehyde (MDA) and serum nitric oxide (NO). Homeostasis model assessment of insulin resistance values were calculated from fasting glucose and insulin measures according to the formula (fasting glucose [mmol/ 1] x fasting insulin $[\mu \mathrm{U} / \mathrm{ml}] / \mathbf{2 2 . 5})$.

Table (1) shows comparison between the three studied groups regarding Fasting Glucose, Insulin and HBA1c\%. Fasting glucose in control group ranged from 3.905.40 with mean value $\mathbf{4 . 6 8} \pm \mathbf{0 . 5 6}$, in group I ranged from 8.50- 16 with mean value 11.29 $\pm \mathbf{2 . 0 1}$, while in group II ranged from 10-17 with mean value 13.86 $\pm \mathbf{1 . 9 7}$.

Insulin in control group ranged from 42-80 with mean value 62.10 \pm 11.20 , in group I ranged from 53.80-175 with mean value $\mathbf{1 0 8 . 8 5} \pm \mathbf{2 9 . 0 4}$, while in group II ranged from 112-345 with mean value $\mathbf{1 9 8 . 2 6} \pm 66.96$.

HBA1c \% in control group ranged from 3.90-6.20 with mean value 5.07 $\pm \mathbf{0 . 7 9}$, in group I ranged from 6.80-11.20 with mean value 8.47 \pm 1.14 , while in group II ranged from 7.80-11.50 with mean value $9.88 \pm 1.07$. There was statistical significant difference between the three studied groups regarding Fasting Glucose, Insulin and HBA1c \% (P= 0.0001).

Table (2) shows comparison between the three studied groups regarding HOMA level. Beta cell function \%(HOMA2 \% B) in control group ranged from 83.20-194.70 with mean value 124.09 $\pm \mathbf{3 3 . 0 7}$, in group I ranged from $\mathbf{1 8 . 0} \mathbf{- 5 7 . 2 0}$ with mean value $\mathbf{3 6} \pm \mathbf{1 0 . 3 3}$, while in group II ranged from 18.50-66.50 with mean value 41.83 \pm 9.67 .Insulin sensitivity (HOMA2\% $\mathrm{S}$ ) in control group ranged from 71.60136.10 with mean value $\mathbf{9 1 . 1 0} \pm \mathbf{1 9 . 4 4}$, in group I ranged from $\mathbf{2 2 . 5 0 - 8 3 . 5 0}$ with mean value 44.52 $\pm \mathbf{1 3 . 9 7}$, while in group II ranged from $\mathbf{1 0 . 0 0}$-41.60 with mean value 23.41 \pm 8.21. Insulin resistance(HOMA2 IR) in control group ranged from 0.70-1.40 with mean value 1.13 $\pm \mathbf{0 . 2 2}$, in group I ranged from 1.20-4.40 with mean value $\mathbf{2 . 4 5} \pm \mathbf{0 . 7 5}$, while in group II ranged from $\mathbf{2 . 4 0 - 1 0 . 0 0}$ with mean value $4.89 \pm \mathbf{1 . 9 8}$.

There was statistical significant difference between the three studied groups regarding HOMA level $(\mathbf{P}=\mathbf{0 . 0 0 0 1})$. 
Table (1): Comparison between the three studied groups regarding Fasting Glucose, Insulin and HBA1c \%.

\begin{tabular}{|c|c|c|c|c|c|c|c|}
\hline Item & Group & $\begin{array}{c}\text { Mea } \\
\text { n }\end{array}$ & \pm S.D. & Min. & Max. & ANOVA & $\mathbf{p}$ \\
\hline \multirow{3}{*}{$\begin{array}{l}\text { Fasting } \\
\text { Glucose } \\
(\mathrm{mmol} /)\end{array}$} & Control group & 4.68 & 0.56 & 3.90 & 5.40 & 93.320 & $0.0001 * *$ \\
\hline & $\begin{array}{l}\text { Group I (DM } \\
\text { without } \\
\text { Nephropathy) }\end{array}$ & 11.29 & 2.01 & 8.50 & 16.00 & & \\
\hline & $\begin{array}{l}\text { Group II (DM } \\
\text { with } \\
\text { Nephropathy) }\end{array}$ & 13.86 & 1.97 & 10.00 & 17.00 & & \\
\hline \multirow{3}{*}{$\begin{array}{l}\text { Insulin } \\
(\mathrm{pmol} / \mathrm{L})\end{array}$} & Control group & 62.10 & 11.20 & 42.00 & 80.00 & 44.274 & $0.0001 * *$ \\
\hline & $\begin{array}{l}\text { Group I (DM } \\
\text { without } \\
\text { Nephropathy) } \\
\end{array}$ & 108.85 & 29.04 & 53.80 & 175.00 & & \\
\hline & $\begin{array}{l}\text { Group II (DM } \\
\text { with } \\
\text { Nephropathy) }\end{array}$ & 198.26 & 66.96 & 112.00 & 345.00 & & \\
\hline \multirow[t]{3}{*}{ HBA1c (\%) } & Control group & 5.07 & 0.79 & 3.90 & 6.20 & 78.518 & $0.0001 * *$ \\
\hline & $\begin{array}{l}\text { Group I (DM } \\
\text { without } \\
\text { Nephropathy) }\end{array}$ & 8.47 & 1.14 & 6.80 & 11.20 & & \\
\hline & $\begin{array}{l}\text { Group II (DM } \\
\text { with } \\
\text { Nephropathy) }\end{array}$ & 9.88 & 1.07 & 7.80 & 11.50 & & \\
\hline
\end{tabular}

Table (2): Comparison between the three studied groups regarding HOMA level.

\begin{tabular}{|c|c|c|c|c|c|c|c|}
\hline Item & Group & Mean & \pm S.D. & Min. & Max. & ANOVA & p \\
\hline \multirow[t]{3}{*}{$\begin{array}{l}\text { Beta cell function } \\
\%(\text { HOMA2 \%B) }\end{array}$} & $\begin{array}{l}\text { Control } \\
\text { group }\end{array}$ & 124.09 & 33.07 & 83.20 & 194.70 & 145.489 & $\begin{array}{c}\mathbf{0 . 0 0 0 1} \\
* *\end{array}$ \\
\hline & $\begin{array}{l}\text { Group I (DM } \\
\text { without } \\
\text { Nephropathy) }\end{array}$ & 36.00 & 10.33 & 18.00 & 57.20 & & \\
\hline & $\begin{array}{l}\text { Group II } \\
\text { (DM with } \\
\text { Nephropathy) }\end{array}$ & 41.83 & 9.67 & 18.50 & 66.50 & & \\
\hline \multirow[t]{3}{*}{$\begin{array}{l}\text { Insulin sensitivity } \\
\text { (HOMA2\%S) }\end{array}$} & $\begin{array}{l}\text { Control } \\
\text { group }\end{array}$ & 91.10 & 19.44 & 71.60 & 136.10 & 112.036 & $\begin{array}{c}\mathbf{0 . 0 0 0 1} \\
* *\end{array}$ \\
\hline & $\begin{array}{l}\text { Group I (DM } \\
\text { without } \\
\text { Nephropathy) }\end{array}$ & 44.52 & 13.97 & 22.50 & 83.50 & & \\
\hline & $\begin{array}{l}\text { Group II } \\
\text { (DM with } \\
\text { Nephropathy) }\end{array}$ & 23.41 & 8.21 & 10.00 & 41.60 & & \\
\hline \multirow{3}{*}{$\begin{array}{l}\text { Insulin } \\
\text { resistance(HOMA2 } \\
\text { IR) }\end{array}$} & $\begin{array}{l}\text { Control } \\
\text { group }\end{array}$ & 1.13 & 0.22 & 0.70 & 1.40 & 39.806 & $\begin{array}{c}\mathbf{0 . 0 0 0 1} \\
* *\end{array}$ \\
\hline & $\begin{array}{l}\text { Group I (DM } \\
\text { without } \\
\text { Nephropathy) }\end{array}$ & 2.45 & 0.75 & 1.20 & 4.40 & & \\
\hline & $\begin{array}{l}\text { Group II } \\
\text { (DM with } \\
\text { Nephropathy) }\end{array}$ & 4.89 & 1.98 & 2.40 & 10.00 & & \\
\hline
\end{tabular}

Table (3) shows comparison between the three studied groups regarding sICAM-1, Nitric oxide and MDA.

sICAM-1 in control group ranged from 149-309 with mean value $\mathbf{6 4 . 7 0} \pm \mathbf{2 2 8 . 8 0}$, in group I ranged from $\mathbf{1 7 5 . 0} \mathbf{- 3 4 5 . 0}$ with mean value $\mathbf{2 8 4 . 1 2} \pm \mathbf{4 9 . 6 9}$, while in group II ranged from 180.0 - 355.0 with mean value $284.18 \pm 47.01$. 
Nitric oxide in control group ranged from $\mathbf{2 2 . 4 0}-\mathbf{4 6 . 0 0}$ with mean value 33.47 \pm 8.25, in group I ranged from $29.0-54.0$ with mean value $35.38 \pm 4.40$, while in group II ranged from $30.0-\mathbf{5 4 . 0}$ with mean value $\mathbf{3 6 . 6 2} \pm \mathbf{4 . 1 6}$.

MDA in control group ranged from $\mathbf{0 . 2 5 - 1 . 4 5}$ with mean value $\mathbf{0 . 9 8} \pm \mathbf{0 . 3 7}$, in group I ranged from 1.50-4.90 with mean value $\mathbf{2 . 7 1} \pm \mathbf{1 . 0 1}$, while in group II ranged from 1.60-4.90 with mean value $3 \pm \mathbf{1 . 0 8}$.

There was statistical significant difference between the three studied groups regarding sICAM-1 and MDA $(\mathrm{P}=\mathbf{0 . 0 0 0 1})$, while there was no statistical significant difference regarding Nitric oxide $(\mathrm{P}=\mathbf{0 . 0 0 0 1})$.

Table (3): Comparison between the three studied groups regarding sICAM-1,

Nitric oxide and MDA.

\begin{tabular}{|c|c|c|c|c|c|c|c|}
\hline Item & Group & Mean & \pm S.D. & Min. & Max. & ANOVA & $\mathbf{p}$ \\
\hline \multirow{3}{*}{$\begin{array}{l}\text { sICAM-1 } \\
\text { (ng/ml) }\end{array}$} & Control group & 228.80 & 64.70 & 149.00 & 309.00 & 5.213 & 0.008* \\
\hline & $\begin{array}{l}\text { Group I (DM without } \\
\text { Nephropathy) }\end{array}$ & 284.12 & 49.69 & 175.00 & 345.00 & & \\
\hline & $\begin{array}{l}\text { Group II (DM with } \\
\text { Nephropathy) }\end{array}$ & 284.18 & 47.01 & 180.00 & 355.00 & & \\
\hline \multirow{3}{*}{$\begin{array}{l}\text { Nitric oxide } \\
(\mu \mathrm{mol} / \mathrm{L})\end{array}$} & Control group & 33.47 & 8.25 & 22.40 & 46.00 & 1.681 & 0.193 \\
\hline & $\begin{array}{l}\text { Group I (DM without } \\
\text { Nephropathy) }\end{array}$ & 35.38 & 4.40 & 29.00 & 54.00 & & \\
\hline & $\begin{array}{l}\text { Group II (DM with } \\
\text { Nephropathy) }\end{array}$ & 36.62 & 4.16 & 30.00 & 54.00 & & \\
\hline \multirow{3}{*}{$\begin{array}{l}\text { MDA } \\
(\mu \mathrm{mole} / \mathrm{L})\end{array}$} & Control group & 0.98 & 0.37 & 0.25 & 1.45 & 16.470 & 0.0001* \\
\hline & $\begin{array}{l}\text { Group I (DM without } \\
\text { Nephropathy) }\end{array}$ & 2.71 & 1.01 & 1.50 & 4.90 & & \\
\hline & $\begin{array}{l}\text { Group II (DM with } \\
\text { Nephropathy) }\end{array}$ & 3.00 & 1.08 & 1.60 & 4.90 & & \\
\hline
\end{tabular}

Table (4) shows comparison between the three studied groups regarding GSH and SOD.

GSH in control group ranged from 13.0 -14.0 with mean value 13.90 $\pm \mathbf{0 . 5 3}$, in group I ranged from 12.80-14.90with mean value $\mathbf{1 3 . 5 4} \pm \mathbf{0 . 6 4}$, while in group II ranged from 12.70-14.80 with mean value $13.44 \pm 0.63$.

SOD in control group ranged from 8.80-12.20 with mean value 10.59 $\pm \mathbf{1 . 1 2}$, in group I ranged from 8.90-14.60 with mean value 11.91 $\pm \mathbf{1 . 8 4}$, while in group II ranged from $11.90-20.00$ with mean value $14.76 \pm 2.12$.

There was statistical significant difference between the three studied groups regarding SOD $(\mathrm{P}<\mathbf{0 . 0 5})$, while there was no statistical significant difference regarding GSH (P> 0.05).

Table (4): Comparison between the three studied groups regarding GSH and

SOD.

\begin{tabular}{|c|c|c|c|c|c|c|c|}
\hline Item & Group & Mean & \pm S.D. & Min. & Max. & ANOVA & p \\
\hline \multirow{3}{*}{$\begin{array}{l}\text { GSH } \\
(\mathrm{ng} / \mathrm{ml})\end{array}$} & Control group & 13.90 & $\mathbf{0 . 5 3}$ & 13.00 & 14.90 & 2.129 & 0.126 \\
\hline & $\begin{array}{l}\text { Group I (DM } \\
\text { without } \\
\text { Nephropathy) }\end{array}$ & 13.54 & 0.64 & 12.80 & 14.90 & & \\
\hline & $\begin{array}{l}\text { Group II (DM } \\
\text { with } \\
\text { Nephropathy) }\end{array}$ & 13.44 & 0.63 & 12.70 & 14.80 & & \\
\hline \multirow{3}{*}{$\begin{array}{l}\text { SOD } \\
\text { activity } \\
(\mathbf{u} / \mathrm{ml})\end{array}$} & Control group & 10.59 & 1.12 & 8.80 & 12.20 & 28.165 & $.0001 *$ \\
\hline & $\begin{array}{l}\text { Group I (DM } \\
\text { without } \\
\text { Nephropathy) }\end{array}$ & 11.91 & 1.84 & 8.90 & 14.60 & & \\
\hline & $\begin{array}{l}\text { Group II (DM } \\
\text { with } \\
\text { Nephropathy) }\end{array}$ & 14.76 & 2.12 & 11.90 & 20.00 & & \\
\hline
\end{tabular}


Table (5) shows comparison between the three studied groups regarding lipid profile.

S. Cholesterol in control group ranged from 150-190 with mean value 14.3 \pm 170 , in group I ranged from 150.0 -290.0 with mean value $\mathbf{2 2 2 . 7} \pm \mathbf{4 2 . 5}$, while in group II ranged from $240.0-370.0$ with mean value $305.7 \pm 40.9$.

S. Trig in control group ranged from $115.0-152.0$ with mean value $139.2 \pm 11.6$, in group I ranged from $\mathbf{1 1 5 . 0}$ - $\mathbf{2 9 0 . 0}$ with mean value $\mathbf{1 8 6 . 1} \pm \mathbf{3 3 . 7}$, while in group II ranged from $165.0-325.0$ with mean value $248.7 \pm 44.1$.

HDL in control group ranged from 40.0 - 69.0 with mean value 55.5 $\pm \mathbf{1 0 . 2}$, in group I ranged from 37.0 - 69.0 with mean value 47.8 \pm 8.5, while in group II ranged from 36.0 - 56.0 with mean value 45.3 \pm 5.2. $L D L$ in control group ranged from 56.0 - 99.0 with mean value $\mathbf{8 6 . 7} \pm \mathbf{1 5 . 0}$, in group I ranged from $\mathbf{5 2 . 8 0 - 2 1 0 . 0}$ with mean value $\mathbf{1 3 7 . 7} \pm \mathbf{4 2 . 3}$, while in group II ranged from $\mathbf{1 3 5 . 0}$ - $\mathbf{2 8 1 . 0}$ with mean value 210.6 \pm 40.5.

There was statistical significant difference between the three studied groups regarding lipid profile ( $\mathrm{P}=\mathbf{0 . 0 0 0 1}$ for S. Cholesterol, S. Trig. and LDL.) And ( $\mathrm{P}=$ $\mathbf{0 . 0 0 1}$ for HDL)

Table (5): Comparison between the three studied groups regarding lipid profile.

\begin{tabular}{|c|c|c|c|c|c|c|c|}
\hline Item & Group & Mean & $\begin{array}{c} \pm \text { S.D } \\
.\end{array}$ & Min. & Max. & $\begin{array}{c}\text { ANO } \\
\text { VA }\end{array}$ & $\mathbf{p}$ \\
\hline \multirow{3}{*}{$\begin{array}{l}\text { S. } \\
\text { Cholesterol( } \\
\text { mg/dl) }\end{array}$} & Control group & 170.0 & 14.3 & $\begin{array}{c}150.0 \\
0\end{array}$ & $\begin{array}{c}190.0 \\
0\end{array}$ & 62.538 & 0.0001* \\
\hline & $\begin{array}{l}\text { Group I (DM } \\
\text { without } \\
\text { Nephropathy) }\end{array}$ & 222.7 & 42.5 & $\begin{array}{c}150.0 \\
0\end{array}$ & $\begin{array}{c}290.0 \\
0\end{array}$ & & \\
\hline & $\begin{array}{l}\text { Group II (DM with } \\
\text { Nephropathy) }\end{array}$ & 305.7 & 40.9 & $\begin{array}{c}240.0 \\
0\end{array}$ & $\begin{array}{c}370.0 \\
0\end{array}$ & & \\
\hline \multirow[t]{3}{*}{$\begin{array}{l}\text { S. } \\
\text { Trig(mg/dl) }\end{array}$} & Control group & 139.2 & 11.6 & $\begin{array}{c}115.0 \\
0\end{array}$ & $\begin{array}{c}152.0 \\
0\end{array}$ & 43.678 & 0.0001* \\
\hline & $\begin{array}{l}\text { Group I (DM } \\
\text { without } \\
\text { Nephropathy) }\end{array}$ & 186.1 & 33.7 & $\begin{array}{c}115.0 \\
0\end{array}$ & $\begin{array}{c}290.0 \\
0\end{array}$ & & \\
\hline & $\begin{array}{l}\text { Group II (DM with } \\
\text { Nephropathy) }\end{array}$ & 248.7 & 44.1 & $\begin{array}{c}165.0 \\
0\end{array}$ & $\begin{array}{c}325.0 \\
0\end{array}$ & & \\
\hline \multirow[t]{3}{*}{ HDL(mg/dl) } & Control group & 55.5 & 10.2 & 40.00 & 69.00 & 7.165 & 0.001* \\
\hline & $\begin{array}{l}\text { Group I (DM } \\
\text { without } \\
\text { Nephropathy) }\end{array}$ & 47.8 & 8.5 & 37.00 & 69.00 & & \\
\hline & $\begin{array}{l}\text { Group II (DM with } \\
\text { Nephropathy) }\end{array}$ & 45.3 & 5.2 & 36.00 & 56.00 & & \\
\hline \multirow[t]{3}{*}{ LDL(mg/dl) } & Control group & 86.7 & 15.0 & 56.00 & 99.00 & 51.077 & 0.0001* \\
\hline & $\begin{array}{l}\text { Group I (DM } \\
\text { without } \\
\text { Nephropathy) }\end{array}$ & 137.7 & 42.3 & 52.80 & $\begin{array}{c}210.0 \\
0\end{array}$ & & \\
\hline & $\begin{array}{l}\text { Group II (DM with } \\
\text { Nephropathy) }\end{array}$ & 210.6 & 40.5 & $\begin{array}{c}135.0 \\
0\end{array}$ & $\begin{array}{c}281.0 \\
0\end{array}$ & & \\
\hline
\end{tabular}

Table (6) shows comparison between the three studied groups regarding kidney function. S.

Creatinine in control group ranged from $\mathbf{0 . 5 5 - 1 . 1 5}$ with mean value $\mathbf{0 . 8 3} \pm \mathbf{0 . 1 9}$, in group I ranged from $\mathbf{0 . 5 5 - 1 . 4 0}$ with mean value $\mathbf{1 . 0 8} \pm \mathbf{0 . 2 3}$, while in group II ranged from $1.25-3.00$ with mean value $1.66 \pm 0.38$. B. Urea in control group ranged from 16.0 - 35.0 with mean value 26.20 \pm 6.99, in group I ranged from 16.0-43.0 with mean value $33.91 \pm 6.90$, while in group II ranged from $\mathbf{3 5 . 0}-\mathbf{7 5 . 0}$ with mean value $51.03 \pm 10.58$. 
Urine Creatinine in control group ranged from $\mathbf{2 4 0 . 0}-\mathbf{3 8 0 . 0}$ with mean value $\mathbf{3 0 1} \pm \mathbf{4 8 . 9 2}$, in group I ranged from 255.0 - 375.0 with mean value 306.56 $\pm \mathbf{2 7 . 0 6}$, while in group II ranged from 235.0 - 358.0 with mean value 296.65 \pm 33.71 .

Urine microalbumen in control group ranged from $\mathbf{6 . 0}-\mathbf{1 1 . 0}$ with mean value 8.0 $\pm \mathbf{1 . 6 0}$, in group I ranged from 5.60-10.70 with mean value $8.21 \pm 1.35$, while in group II ranged from $35.0-230.0$ with mean value $86.03 \pm 46.43$.

A/C Ratio Creatinine in control group ranged from 22.60-29.30 with mean value 26.54 $\pm \mathbf{2 . 4 6}$, in group I ranged from $18.50-30.10$ with mean value $26.74 \pm 3.42$, while in group II ranged from 116.7 -978.7 with mean value $\mathbf{3 0 4 . 8 8} \pm \mathbf{1 9 7 . 1 0}$. There was statistical significant difference between the three studied groups regarding $\mathrm{S}$. Creatinine, B. Urea, Urine microalbumen and A/C Ratio Creatinine $(\mathrm{P}<\mathbf{0 . 0 5})$, while there was no statistical significant difference regarding Urine Creatinine $(\mathbf{P}>\mathbf{0 . 0 5})$.

Table (6): Comparison between the three studied groups regarding kidney function.

\begin{tabular}{|c|c|c|c|c|c|c|c|}
\hline Item & Group & Mean & \pm S.D. & Min. & Max. & ANOVA & p \\
\hline \multirow[t]{3}{*}{ S. Creatinine(mg/dl) } & $\begin{array}{l}\text { Control } \\
\text { group }\end{array}$ & 0.83 & 0.19 & 0.55 & 1.15 & 46.458 & 0.0001* \\
\hline & $\begin{array}{l}\text { Group I (DM } \\
\text { without } \\
\text { Nephropathy) }\end{array}$ & 1.08 & 0.23 & 0.55 & 1.40 & & \\
\hline & $\begin{array}{l}\text { Group II } \\
\text { (DM with } \\
\text { Nephropathy) }\end{array}$ & 1.66 & 0.38 & 1.25 & 3.00 & & \\
\hline \multirow[t]{3}{*}{ B. Urea(mg/dl) } & $\begin{array}{l}\text { Control } \\
\text { group }\end{array}$ & 26.20 & 6.99 & 16.00 & 35.00 & 47.910 & 0.0001* \\
\hline & $\begin{array}{l}\text { Group I (DM } \\
\text { without } \\
\text { Nephropathy) }\end{array}$ & 33.91 & 6.90 & 16.00 & 43.00 & & \\
\hline & $\begin{array}{l}\text { Group II } \\
\text { (DM with } \\
\text { Nephropathy) }\end{array}$ & 51.03 & 10.58 & 35.00 & 75.00 & & \\
\hline \multirow[t]{3}{*}{$\begin{array}{l}\text { Urine } \\
\text { Creatinine(mg/dl) }\end{array}$} & $\begin{array}{l}\text { Control } \\
\text { group }\end{array}$ & 301.00 & 48.92 & 240.00 & 380.00 & .754 & 0.474 \\
\hline & $\begin{array}{l}\text { Group I (DM } \\
\text { without } \\
\text { Nephropathy) }\end{array}$ & 306.56 & 27.06 & 255.00 & 375.00 & & \\
\hline & $\begin{array}{l}\text { Group II } \\
\text { (DM with } \\
\text { Nephropathy) }\end{array}$ & 296.65 & 33.71 & 235.00 & 358.00 & & \\
\hline \multirow[t]{3}{*}{$\begin{array}{l}\text { Urine } \\
\text { microalbumen(mg/dl) }\end{array}$} & $\begin{array}{l}\text { Control } \\
\text { group }\end{array}$ & 8.00 & 1.60 & 6.00 & 11.00 & 61.242 & $0.0001 *$ \\
\hline & $\begin{array}{l}\text { Group I (DM } \\
\text { without } \\
\text { Nephropathy) }\end{array}$ & 8.21 & 1.35 & 5.60 & 10.70 & & \\
\hline & $\begin{array}{l}\text { Group II } \\
\text { (DM with } \\
\text { Nephropathy) }\end{array}$ & 86.03 & 46.43 & 35.00 & 230.00 & & \\
\hline \multirow[t]{3}{*}{$\begin{array}{l}\text { A/C Ratio } \\
\text { (mg/gcreatinine) }\end{array}$} & $\begin{array}{l}\text { Control } \\
\text { group }\end{array}$ & 26.54 & 2.46 & 22.60 & 29.30 & 43.398 & 0.0001* \\
\hline & $\begin{array}{l}\text { Group I (DM } \\
\text { without } \\
\text { Nephropathy) }\end{array}$ & 26.74 & 3.42 & 18.50 & 30.10 & & \\
\hline & $\begin{array}{l}\text { Group II } \\
\text { (DM with } \\
\text { Nephropathy) }\end{array}$ & 304.88 & 197.10 & 116.70 & 978.70 & & \\
\hline
\end{tabular}

Table (7) shows correlation between different studied markers, it was found that there was a positive significant correlation between SICAM-1 and all other variables include (insulin, nitric oxide, MDA and SOD activity), also it was found that there 
was a positive significant correlation between insulin and MDA and SOD activity.

Nitric oxide has a significant positive correlation with SOD activity.

Table (7): Correlation between different studied markers.

\begin{tabular}{|c|c|c|c|c|c|c|}
\hline \multicolumn{2}{|l|}{ Item } & \multirow{2}{*}{$\begin{array}{c}\text { sICAM-1 } \\
\text { ng/ml }\end{array}$} & \multirow{2}{*}{$\begin{array}{l}\begin{array}{l}\text { Insulin } \\
\text { pmol/L }\end{array} \\
\mathbf{0 . 3 0 5}^{\text {** }}\end{array}$} & \multirow{2}{*}{$\begin{array}{c}\begin{array}{c}\text { Nitric } \\
\text { oxide } \\
\mu \mathrm{mol} / \mathrm{L}\end{array} \\
\mathbf{0 . 3 5 0}^{* * *}\end{array}$} & \multirow{2}{*}{$\begin{array}{c}\begin{array}{c}\text { MDA } \\
\text { Mmole/ } \\
\text { L }\end{array} \\
\mathbf{0 . 4 3 2}^{* *}\end{array}$} & \multirow{2}{*}{$\begin{array}{c}\begin{array}{c}\text { SOD } \\
\text { activity } \\
\text { u/ml }\end{array} \\
\mathbf{0 . 2 4 3}^{*}\end{array}$} \\
\hline $\begin{array}{l}\text { SICAM-1 } \\
\text { (ng/ml) }\end{array}$ & $\begin{array}{l}\text { Pearson } \\
\text { Correlation }\end{array}$ & & & & & \\
\hline & $\mathbf{p}$ & & $\mathbf{0 . 0 0 7}$ & $\mathbf{0 . 0 0 2}$ & 0.000 & $\mathbf{0 . 0 3 2}$ \\
\hline \multirow[t]{2}{*}{$\begin{array}{l}\text { Insulin } \\
(\mathrm{pmol} / \mathrm{L})\end{array}$} & $\begin{array}{l}\text { Pearson } \\
\text { Correlation }\end{array}$ & $0.305^{* *}$ & - & 0.197 & $0.577^{* * *}$ & $\mathbf{0 . 7 3 0}^{* *}$ \\
\hline & p & 0.007 & & 0.084 & 0.000 & 0.000 \\
\hline \multirow{2}{*}{$\begin{array}{l}\text { Nitric } \\
\text { oxide } \\
(\mu \mathrm{mol} / \mathrm{L})\end{array}$} & $\begin{array}{l}\text { Pearson } \\
\text { Correlation }\end{array}$ & $0.350 *$ & 0.197 & - & 0.109 & $0.285^{*}$ \\
\hline & $\mathbf{p}$ & 0.002 & 0.084 & & 0.342 & 0.011 \\
\hline \multirow[t]{2}{*}{$\begin{array}{l}\text { MDA } \\
(\mu \text { mole/L })\end{array}$} & $\begin{array}{l}\text { Pearson } \\
\text { Correlation }\end{array}$ & $0.432^{* * *}$ & $0.577^{* *}$ & 0.109 & - & $0.467^{* * *}$ \\
\hline & $\mathbf{p}$ & 0.000 & 0.000 & 0.342 & & 0.000 \\
\hline \multirow{2}{*}{$\begin{array}{l}\text { SOD } \\
\text { activity } \\
\text { (u/ml) }\end{array}$} & $\begin{array}{l}\text { Pearson } \\
\text { Correlation }\end{array}$ & $\mathbf{0 . 2 4 3}^{*}$ & $\mathbf{0 . 7 3 0}^{* * *}$ & $\mathbf{0 . 2 8 5}^{*}$ & $0.467^{* *}$ & - \\
\hline & $\mathbf{p}$ & 0.032 & $\mathbf{0 . 0 0 0}$ & 0.011 & 0.000 & \\
\hline \multicolumn{7}{|c|}{ **. Correlation is significant at the 0.01 level (2-tailed). } \\
\hline & *. Corr & ion is signif & t at the 0 & vel (2-tai & & \\
\hline
\end{tabular}

Table (8), show the correlation between sICAM-1 and urine microalbumin and blood urea, it was found that there was a positive significant correlation between sICAM-1 and both blood urea and urine microalbumin urea.

Table (8): Correlation between sICAM-1 and urine microalbumin and blood urea.

\begin{tabular}{|l|c|c|}
\hline sICAM-1 \# & Person correlation & P \\
\hline Blood urea & 0.292 & $0.009^{*}$ \\
\hline Urine microalbumin urea & 0.277 & $0.0133^{*}$ \\
\hline
\end{tabular}

\section{DISCUSSION}

In molecular biology, intercellular adhesion molecules (ICAMs) and vascular cell adhesion molecule-1 (VCAM-1) are part of the immunoglobulin superfamily. They are important in inflammation, immune responses and in intracellular signalling events. The ICAM family consists of five members, designated ICAM-1 to ICAM-5. They are known to bind to leucocyte integrins CD11/CD18 such as Lymphocyte function antigen-1(LFA-1) and Macrophage-1 antigen, during inflammation and in immune responses. In addition, ICAMs may exist in soluble forms in human plasma, due to activation and proteolysis mechanisms at cell surfaces.

Mammalian intercellular adhesion molecules include: ICAM-1, ICAM2, ICAM3 ICAM4 ICAM5 (14).

Much of the morbidity associated with type 2 diabetes is not a direct consequence of the disease itself, but results from the related complications (15).

In the programmed studied by The World Health Organization, 2006 for 
the control of type 2 diabetes, it was found that $\mathbf{8 \%}$ have already nephropathy; since many people with type 2 diabetes are diagnosed in a later stage of the disease, changes are higher in their kidneys; it seems to be already damaged.

Critical proteins expressed by endothelial cells that bind the inflammatory cells are the cell adhesion molecules.

In accordance to our results there appears to be a strong relationship between sICAM-1 (intercellular adhesion molecule-1) and diabetic nephropathy. It has been shown that inflammation has a role in the development of diabetic nephropathy (16), so the high levels of sICAM-1 may play a role in the development of nephropathy in patients with type $\mathbf{2}$ diabetes mellitus.

In this work; we focused on metabolic severity of nephropathy according to degree of protein present in urine, this concept is accepted by' De Vriese et al., 2000 (17) who reported that diabetic nephropathy is microvascular complication of longstanding diabetes; the earliest accessible manifestation of this development is the presence of albumin in urine. Ogata et al. (2007) (18) added explanation that with worsen diabetic control, increase probability of nephropathy and increases nephron hyper filtration which results in increase albumin excretion rate; these results clarified that with increase occurrence of albumin in urine which defined as microalbuminuria; consider as a sign of diabetic nephropathy.

Inflammation is associated with the microalbuminuric state, importantly that sICAM-1 has been found to be significantly associated with microalbuminuria in type 2 diabetics as compared with control (19) and these agree with our result which show positive direct correlation between ICAM-1 and microalbuminuria ( $\mathrm{r}$ value $=0.277, \mathrm{P} 0.0133^{*}$ ), so our study show relationship between inflammation and diabetic complication by the strong correlation between ICAM-1 and microalbuminuria.

In the present study, there is significant increase in the insulin resistance in diabetic patients without nephropathy and with nephropathy as compared with control group and also our results show strong direct correlation between ICAM-1 and insulin resistance, this agree with (20), which reported that the increased plasma levels of adhesion molecules found in type $\mathbf{2}$ diabetes are related to insulin resistance and/or compensatory hyperinsulinemia. This finding supports the conclusion that insulin resistance may be a factor contributing to the increase plasma levels of adhesion molecules in the clinical conditions mentioned above.

Insulin resistance is associated with a cluster of metabolic abnormalities and this is referred to as the insulin resistance syndrome or the metabolic syndrome (21).

In our study the metabolic syndrome and type 2 diabetes are associated with endothelial activation, but the mechanisms that underlie these associations are not fully understood. This is agreeing with (22) who reported that ICAM-1 concentration showed significant positive genetic correlations with fasting insulin and homeostasis model assessment of insulin resistance. Thus, ICAM- 1 expression may share common genetic modulation with traits related to insulin resistance. Also this result supported by (23) who reported that the association between ICAM-1 expression and insulin resistance is consistent with clinical evidence relating insulin resistance and 
inflammation. Also there is a strong relationship between sICAM-1 and diabetic nephropathy. Several previous publications agree with our results have shown that soluble adhesion molecules are increased in patients with hypertriglyceridemia, essential hypertension, gestational diabetes and type 2 diabetes. The common element in these disparate clinical syndromes is the presence of resistance to insulinmediated glucose disposal, raising the possibility that the increase in circulating soluble adhesion molecules is secondary to insulin resistance (24).

\section{REFERENCES}

1-"Causes of Diabetes". National Institute of Diabetes and Digestive and Kidney Diseases (NIDDK), June 2014. Retrieved 10 February 2016.

2-American Diabetes Association. Classification and diagnosis of diabetes. Diabetes Care. 2016;39 (Suppl. 1): S13-S22.

3-"About diabetes". World Health Organization. Retrieved 4 April 2014.

4-Pasquel, FJ; Umpierrez, GE (November 2014). "Hyperosmolar hyperglycemic state: a historic review of the clinical presentation, diagnosis, and treatment.". Diabetes Care 37 (11): 3124-31.

5-Atkins RC. (2005): The epidemiology of chronic kidney disease. Kidney Int Suppl. 2005;S14-S18.

6-Burrows NR, Li Y, Geiss LS. (2010): Incidence of treatment for end-stage renal disease among individuals with diabetes in the U.S. continues to decline. Diabetes Care. 2010;33:73-77.

7-Stumvoll M, Goldstein BJ, van Haeften TW. (2005): Type 2 diabetes: principles of pathogenesis and therapy. Lancet. 2005;365:1333-1346.

8-Iseki K, Ikemiya Y, Kinjo K, Iseki C, Takishita S. (2004): Prevalence of high fasting plasma glucose and risk of developing end-stage renal disease in screened subjects in Okinawa, Japan. ClinExpNephrol. 2004;8:250-256.

9-Giacco F, Brownlee M. (2010): Oxidative stress and diabetic complications. Circ Res. 2010;107:1058-1070.

10-Raza, H.; Prabu, K.; Robin, S. and Avadhani, G. (2004): Elevated mitochondrial cytochrome P450 2E1 and glutathione-S- transferase A4-4 in STZ induced diabetic rats: tissuespecific variations and roles in oxidative stress. Diabetes, 53: 185-94.

11-Staunton DE, Marlin SD, Stratowa C, Dustin ML, Springer TA. (1988): Primary structure of ICAM-1 demonstrates interaction between members of the immunoglobulin and integrin supergene families. Cell. 1988;52:925-933.

12-Chow FY, Nikolic-Paterson DJ, Ozols E, Atkins RC, Tesch GH (2005) Intercellular adhesion molecule-1 defiiency is protective against nephropathy in type 2 diabetic $\mathrm{db} / \mathrm{db}$ mice. J Am SocNephrol 16: 17111722.

13-Li JJ, Lee SH, Kim DK, Jin R, Jung DS, Kwak SJ, Kim SH, Han SH, Lee JE, Moon SJ. (2009): Colchicine attenuates inflammatory cell infiltration and extracellular matrix accumulation in diabetic nephropathy. Am J Physiol Renal Physiol. 2009;297:F200-F209.

14-Gahmberg CG, Tolvanen M, Kotovuori P.; 1997:"Leukocyte adhesion--structure and function of human leukocyte beta2-integrins and their cellular ligands". Eur. J. Biochem. 245 (2): 215-32.

15-Shanahan, C.M. (2005): mechanisms of vascular calcification in renal disease. Clin.Nephrol.; 63:146-156. 
16-Chung FU, Shin SJ, Tsai JC et al (2005): Peripheral total and differential leukocyte count in diabetic nephropathy. Diabetes care, 2005; 8:1710-179.

17-De Vriese, A.S., Verbeuren, T.J., Voorde, J. V, Lameire, N.H. and 5. Vanhautte, P.M. (2000): Endothelial dysfunction in diabetes. Br.J. Pharmacol.; 130:963-974

18-Ogata, N., Matsuoka, M., Matsuyama, $\mathrm{K}$, Sltima, C. and Matsumura, $\mathrm{M}$. (2007):Plasma concentration of pigment endothelium derived factor in type 2 diabetic patients with micro vascular complications J. Clin. Endocrinol. Metab.; 92(3): 1176-9.

19-Murakami H, TamasawaN,Matsui J, et al (2001) :Plasma levels of soluble vascular adhesion molecule-1 and cholesterol oxidation product in type $\mathbf{2}$ diabetic patients with nephropathy. J AtherosclerThromb. 8:21-51.

20-Marfella R, Esposito K, Giunta R, Coppola G, De Angelis L, Farzati B, Paolisso G, Giugliano D. (2000): Circulating adhesion molecules in humans: role of hyperglycemia and hyperinsulinemia.

Circulation 101:2247-2251,2000.
21-Ford ES, Giles WH, Dietz WH, (2002): Prevalence of the metabolic syndrome among US adults: findings from the third National Health and Nutrition Examination Survey. J AMA; 16; 287:356-359.

22-Jack W, Kent, Jr., Anthony G. Comuzzie, Michael C. Mahaney, Laura Almasy, David L. Rainwater, John L. Vande Berg, Jean W. MacCluer, and John Blangero (2004): Intercellular adhesion molecule-1 concentration is genetically correlated with insulin । resistance, obesity and HDL concentration in Mexican Americans. Diabetes, vol. 53:2691-2695.

23-Hotamisligil

GS.

(2003): Inflammatory pathways and insulin action. Int J Obes 27:553-555.

24-Cominacini L, FrattaPasini A, Garbin $\mathrm{U}$, et al. (1995): Elevated levels of soluble E-selectin in patients with IDDM and NIDDM: relation to metabolic control. Diabetologia. 38:1122-1124. 